\title{
Paradoxical non-linear response of a Brownian particle
}

\author{
Ralf Eichhorn and Peter Reimann \\ Universität Bielefeld, Fakultät für Physik, 33615 Bielefeld, Germany
}

(Dated: June 16, 2018)

\begin{abstract}
We consider a Brownian particle in a "meandering" periodic potential when the ambient temperature is a periodically or stochastically varying function of time. Though far from equilibrium, the linear response of the particle to an external static force is exactly the same as in the equilibrium case, i.e. for constant temperature. Even more surprising is the non-linear response: the particle slows down and then even starts to move in the direction opposite to the applied force.

PACS numbers: 05.40.-a, 05.60.-k, 02.50.Ey
\end{abstract}

The second law of thermodynamics requires that the response of a system at thermal equilibrium to a static external force is a motion in the direction of that force. In this Letter we address the geometrically constrained motion of a Brownian particle with the remarkable property that, though being far from equilibrium, its linear response to a static force $F$ is exactly the same as in the equilibrium case. In particular, the system is at rest in the absence of the external perturbation $F$ and for sufficiently small (positive or negative) $F$ it moves, as naively expected, in the same direction as the static force. An even more remarkable, genuine non-equilibrium behavior arises in the non-linear response regime: upon increasing $F$ to moderately large (positive or negative) values, the particle slows down and then even starts to move in the direction opposite to the applied force $F$ ! Note that such a response behavior is fundamentally different from so-called absolute negative conductance or mobility [1], characterized by linear and non-linear response properties that are just reciprocal to those described above, and from differential negative mobility 2], where the transport speed decreases with increasing force but never points in the direction opposite to that force. Accordingly, also the underlying physics and, in particular, the considered systems are entirely different. Furthermore, our present case has nothing to do with a so-called ratchet effect [3], where, by definition, the velocity of the Brownian particle is finite even in the absence of an external force, thus keeping the same sign in an entire interval of positive and negative forces $F$, and moreover increasing monotonically as a function of $F$.

The above announced response of a Brownian particle to an external force requires the interplay of two indispensable ingredients: a non-linear dynamics and a source of disequilibrium. Both will be chosen in a way that is conceptually very simple and experimentally easy to realize. As for the non-linear dynamics, we consider an overdamped Brownian particle, whose motion is confined to a quasi-one-dimensional meandering path in the $x-y$ plane, and which is subjected to an externally applied homogeneous force along the $x$-axis, see Fig. 1. Experimentally, such a meandering state space can be realized e.g. by means of light forces [4] or morphologically via lithographic etching methods [5], while the external force can be generated e.g. by electrical or magnetic fields, or by gravitation. The second main ingredient of our model is a source of disequilibrium. Since the heydays of Carnot's machines, one of the simplest examples in this respect is a temperature that periodically switches between two different values, see Fig. 2a. Apart from this "minimal" example we will also admit somewhat more general time dependent temperatures $T(t)$, consisting of a "background" or "floor" temperature $T_{0}$, superimposed by short, but violent temperature "pulses", "spikes", or "outbursts", occurring at random times $t_{i}$ and extending over characteristic time intervals $\tau_{i}$, see Fig. 2b. In analogy to the periodic case, the average interspike distance is denoted by

$$
\tau:=\left\langle t_{i+1}-t_{i}\right\rangle
$$

and the relative duration of the temperature spikes by

$$
\vartheta:=\left\langle\tau_{i}\right\rangle / \tau .
$$

Experimentally, such temperature variations may be realized either directly via heating e.g. with laser pulses or indirectly e.g. by pressure pulses. We also note that such outbursts may not necessarily be caused by thermal noise but may as well be considered as a simplified model for sudden mechanical "strikes", "quakes", "impacts", "eruptions" etc. With a negligibly small "background" temperature $T_{0}$, such a model may then describe a variety of experiments with even quite large "particles" subject to some kind of sporadic violent shaking.

The quantity of central interest is the average particle current along the $x$-axis

$$
\langle\dot{x}\rangle:=\lim _{t \rightarrow \infty} \frac{x(t)}{t} .
$$

For ergodicity reasons, no ensemble average on the right hand side is needed and due to the long time limit, initial conditions do not matter. For the symmetric example in Fig. 1b it is clear that the current $\langle\dot{x}\rangle$ is an odd function of the applied force $F$ and in particular vanishes in the absence of the force. For more general cases like in Fig. 1a, the former property is lost but, as we will show later, one still has $\langle\dot{x}\rangle=0$ for $F=0$. In other words, our system does not exhibit a ratchet effect.

Parametrizing by $q$ the position of the particle along its meandering route (path length), its overdamped Brownian motion can be modeled by the Langevin-equation 
$[3,6]$

$$
\eta \dot{q}(t)=-V^{\prime}(q(t))+\sqrt{2 \eta k_{\mathrm{B}} T(t)} \xi(t),
$$

where $\eta$ denotes the damping coefficient, $k_{\mathrm{B}}$ Boltzmann's constant, and $\xi(t)$ a $\delta$-correlated, unbiased Gaussian noise. The force $-V^{\prime}(q)$ in (4) represents the projection of $\vec{e}_{x} F$ in Fig. 1 along the meandering path at the position parametrized by $q$. Thus $V(q)$ is proportional to $F$. Denoting by $L_{q}$ the natural spatial period along the meandering path one can see from Fig. 1 that increasing the path length $q$ by one period $L_{q}$ is tantamount to increasing the $x$-component of the particle position by one period $L_{x}$ and leaving the $y$-component unchanged. As a first consequence, we can infer that $V\left(q+L_{q}\right)=V(q)-F L_{x}$ and hence

$$
\begin{aligned}
V(q) & =F\left[G(q)-q L_{x} / L_{q}\right] \\
G\left(q+L_{q}\right) & =G(q) .
\end{aligned}
$$

As a second consequence, it follows that the average velocity of actual interest (3) is related to the average velocity $\langle\dot{q}\rangle:=\lim _{t \rightarrow \infty}\{q(t) / t\}$ of the auxiliary dynamics (44) according to

$$
\langle\dot{x}\rangle=\langle\dot{q}\rangle L_{x} / L_{q} .
$$

Note that the periodic function $G(q)$ from (5), (6) is $F$-independent. In Fig. 3, we exemplify its explicit construction for the piecewise linear meandering path from Fig. 1b. Whence one can infer that

$$
\begin{aligned}
& G(q)= \begin{cases}-q\left(1-L_{x} / L_{q}\right) & \text { for }-l_{2}<q \leq 0 \\
q\left(\cos \varphi+L_{x} / L_{q}\right) & \text { for } 0<q \leq l_{1}\end{cases} \\
& G\left(q+l_{1}+l_{2}\right)=G(q) \\
& L_{x}=2\left(l_{2}-l_{1} \cos \varphi\right) \\
& L_{q}=2\left(l_{1}+l_{2}\right) .
\end{aligned}
$$

In order to study the linear response behavior of our system, the deterministic force $-V^{\prime}(q)$ in (4), being $L_{q^{-}}$ periodic according to (5), (6), is expanded into a Fourierseries. Since each summand of this Fourier expansion is proportional to $F$ (cf. Eq. (5) it follows that in leading order $F$ (linear response) the effect in (4) of each of those summands can be considered separately and their individual contributions to the current $\langle\dot{q}\rangle$ can simply be added up. For symmetry reasons one can infer [3] that any summand which exhibits harmonic oscillations as a function of $q$ does not lead to a systematic directed transport, i.e. does not contribute to $\langle\dot{q}\rangle$. It remains the non-oscillating part of $-V^{\prime}(q)$, which according to (51) is given by $F L_{x} / L_{q}$, and hence gives rise to a current $\eta\langle\dot{q}\rangle=F L_{x} / L_{q}$ after averaging out the noise in (4). Exploiting (17) we thus can infer that

$$
\langle\dot{x}\rangle=\left(\frac{L_{x}}{L_{q}}\right)^{2} \frac{F}{\eta}+\mathcal{O}\left(F^{2}\right) .
$$

The same result can also be derived in a mathematically more rigorous way by means of a perturbation expansion in $F$ of the Fokker-Planck equation equivalent to (4). Moreover, it can be extended beyond the so far considered case that $T(t)>0$ for all $t$. The most remarkable property of the linear response behavior (12) is its independence of the temperature $T(t)$ : For strong temperature variations, i.e. far from equilibrium, it is exactly the same as in the equilibrium case when the temperature is constant!

Next we turn to the non-linear response for not too small forces $F$. The simplest case arises when $F$ is so large or the "background temperature" $T_{0}$ is so small that in the auxiliary dynamics (4)-(6) the effect of the fluctuations $\xi(t)$ is negligible between successive temperature spikes, cf. Fig. 2. Furthermore, any temperature outburst is assumed to be so violent, that the distribution of an ensemble of Brownian particles will be completely randomized, i.e. practically uniformly distributed within any interval comparable to the spatial period $L_{q}$. Moreover, during such an outburst, the particles will practically not feel the details of the periodic part $G(q)$ of the potential in (5) but only its systematic "tilt" $-q F L_{x} / L_{q}$. Hence, the average displacement during an outburst of duration $\tau_{i}$ is

$$
\Delta q_{s}\left(\tau_{i}\right)=\tau_{i} F L_{x} /\left(L_{q} \eta\right) .
$$

Between two outbursts, the particles start with a uniform initial probability distribution and then evolve deterministically according to (4) with $\xi(t) \equiv 0$. Focusing on the piecewise linear example from Fig. 3b and Eqs. (8)-(11) and on positive forces $F$, one readily finds that the average net displacement during the time $\Delta t_{i}:=t_{i+1}-t_{i}-\tau_{i}$ between two temperature outbursts amounts to

$$
\begin{aligned}
\Delta q_{0}\left(\Delta t_{i}\right) & =\left[l_{2}^{2}-\left(l_{2}-v_{2} \Delta t_{i}\right)^{2} \Theta\left(l_{2}-v_{2} \Delta t_{i}\right)\right] / L_{q} \\
& -\left[l_{1}^{2}-\left(l_{1}-v_{1} \Delta t_{i}\right)^{2} \Theta\left(l_{1}-v_{1} \Delta t_{i}\right)\right] / L_{q}
\end{aligned}
$$

where $v_{2}:=|F| / \eta$ and $v_{1}:=|F| \cos \varphi / \eta$ are the two deterministic particle speeds associated with the two different slopes of the piecewise linear potential $V(q)$ in Fig. 3b and the Heaviside step functions $\Theta$ account for the fact that the particles stop to move once they have reached a local potential minimum of $V(q)$. The average displacement per temperature outburst then follows as $\left\langle\Delta q_{s}\left(\tau_{i}\right)+\Delta q_{0}\left(\Delta t_{i}\right)\right\rangle$. Dividing by the average interspike distance from (11) yields $\langle\dot{q}\rangle$. With (2), (7), (13) and taking into account that $\langle\dot{x}\rangle$ is obviously an odd function of $F$, we finally obtain

$$
\langle\dot{x}\rangle=F \frac{L_{x}}{L_{q}}\left[\frac{\vartheta}{\eta} \frac{L_{x}}{L_{q}}+\frac{\left\langle\Delta q_{0}\left(t_{i+1}-t_{i}-\tau_{i}\right)\right\rangle}{|F| \tau}\right] .
$$

For random temperature bursts, the average $\left\langle\Delta q_{0}\left(t_{i+1}-\right.\right.$ $\left.\left.t_{i}-\tau_{i}\right)\right\rangle$ has to be evaluated according to their specific statistical properties. In many cases, one expects that the result will be well approximated by $\Delta q_{0}\left(\left\langle t_{i+1}-t_{i}-\tau_{i}\right\rangle\right)=$ $\Delta q_{0}([1-\vartheta] \tau)$, i.e.

$$
\langle\dot{x}\rangle=F \frac{L_{x}}{L_{q}}\left[\frac{\vartheta}{\eta} \frac{L_{x}}{L_{q}}+\frac{\Delta q_{0}([1-\vartheta] \tau)}{|F| \tau}\right] .
$$


For periodic bursts, Eqs. (15) and (16) are even strictly equivalent.

For small $F$, one readily recovers the correct linear response behavior (12) from (15), though this small- $F$ regime was originally not included in our considerations above. Next we turn to the case that $|F|$ is sufficiently large that the $\Theta$-functions in (15) vanish. Focusing on the case $l_{1}>l_{2}$ in Fig. 3, this amounts to the condition $(1-\vartheta) \tau|F| \cos \varphi / \eta>l_{1}$, and (15) with (11), (14) then takes the form

$$
\langle\dot{x}\rangle=F \frac{L_{x}}{L_{q}}\left[\frac{\vartheta}{\eta} \frac{L_{x}}{L_{q}}-\frac{l_{1}-l_{2}}{2|F| \tau}\right] .
$$

For sufficiently short relative pulse durations $\vartheta$ we thus expect the existence of an interval of moderately large $|F|$-values with the property that the current $\langle\dot{x}\rangle$ will be positive for negative $F$ and vice versa!

In Figs. 4 and 5, results of numerical simulations are presented, exhibiting a fairly good agreement with the approximation (15). In particular, both the predicted linear response and the paradoxical non-linear response behavior are confirmed. While for the filled circles the assumptions made in the derivation of the approximation (15) are apparently quite well satisfied, for the open circles there are notable deviations. As mentioned above, for the periodic $T(t)$ in Fig. 4 the approximations (15) and (16) coincide, whereas for the stochastic $T(t)$ in Fig. 5 the more complicated expression (15) is clearly superior.

Finally, we turn to a heuristic discussion of the general conditions under which the above paradoxical non-linear response behavior is expected. Taking into account the obvious constraints $0<\varphi<\pi / 2, l_{2} / 2-l_{1} \cos \varphi>0$ in Fig. 3 and $l_{1}>l_{2},(1-\vartheta) \tau|F| \cos \varphi / \eta>l_{1}$ [see above Eq. [17] ] one can infer that a necessary condition to get a current opposite to $F$ in (17) is $\vartheta<1 / 3$. On the other hand, the shorter the relative duration $\vartheta$ of the temperature bursts in (17) are, the more pronounced the effect will be. In particular, for $\vartheta \rightarrow 0$ [i.e. $\delta$-spikes in Fig. 2] the current $\langle\dot{x}\rangle$ in (17) remains opposite to $F$ for arbitrarily large $|F|$. So far we have assumed, that each temperature burst results in an almost uniform particle randomization within any period $L_{q}$. In the opposite case, i.e. for not so violent bursts, the distribution will remain notably peaked around the local potential minima of $V(q)$, leading to a reduced displacement opposite to $F$ during the subsequent relaxation period till the next burst. In other words, the effect of interest diminishes with decreasing intensity of the temperature bursts, and obviously disappears altogether when the temperature variations in Fig. 2 become negligible. Similarly, one sees that our assumption of a negligibly small "background" temperature $T_{0}$, i.e. a deterministic relaxation dynamics in between successive bursts, is optimal in the sense that with increasing the noise intensity $T_{0}$, the effect of interest decreases and finally disappears. These predictions are confirmed by the open circles in Fig. 4, whose deviations from the theoretical solid line can be traced back to the increased $T_{0}$-value for small $F$ and to the reduced $T_{h i g h}$-value for large $F$. Finally, going over from the piecewise linear example in Fig. 1b to the more general case in Fig. 1a leads to an increased deterministic relaxation time towards the local potential minima of the associated auxiliary potentials $V(q)$ and hence again to a reduction of the effect of interest. Notwithstanding, it is clear that a current opposite to an applied force of suitable magnitude will arise whenever the outbursts are sufficiently short and violent, $T_{0}$ is sufficiently small, and if the meandering path is such that a motion along this path with constant velocity will result in a back-and-forth motion when projected along the $x$-axis, whose "backward-segments" are of longer duration than the "forward-segments". Similarly, when going over from the quasi-one-dimensional paths in Fig. 1 (zero "width") to the corresponding meandering structures with a finite "width", we numerically verified (not shown) that the basic qualitative response behavior in Fig. 4 remains unchanged upon extending the width of the black lines in Fig. 3a at least as long as the corners of the structure indicated by the encircled numbers 1 and 4 in Fig. 3a did not yet merge (corresponding to a maximal width of about $0.182 l_{2}$ ).

\section{Acknowledgments}

This work was supported by Deutsche Forschungsgemeinschaft under SFB 613 and RE 1344/3-1 and by the ESF-program STOCHDYN.
[1] T. Y. Banis, I. V. Parshelyunas, and Y. K. Pozhela, Sov. Phys. Semicond. 5, 1727 (1972); V. V. Pavlovich and E. M. Épshteŭn, ibid. 10, 1196 (1976); B. J. Keay et al., Phys. Rev. Lett. 75, 4102 (1995); P. Reimann et al., Europhys. Lett. 45, 545 (1999); R. Eichhorn, P. Reimann, and P. Hänggi, Phys. Rev. Lett. 88, 190601 (2002); B. Cleuren and C. Van den Broeck, Phys. Rev. E 65, 030101(R) (2002).

[2] S. R. White and M. Barma, J. Phys. A 17, 2995 (1984); V. Balakrishnan and C. Van den Broeck, Physica A 217, 1 (1995); G. Cecchi and M. O. Magnasco, Phys. Rev. Lett.
76, 1968 (1996); R. K. P. Zia, E. L. Praestgaard, and O. G. Mourtisen, Am. J. Phys. 70, 384 (2002).

[3] P. Reimann, Phys. Rep. 361, 57 (2002).

[4] L. P. Faucheux et al., Phys. Rev. Lett. 74, 1504 (1995); Q.-H. Wei et al., ibid. 81, 2606 (1998).

[5] W. D. Volkmuth and R. H. Austin, Nature 358, 600 (1992); C.-F. Chou et al., Proc. Natl. Acad. Sci. U.S.A. 96, 13762 (1999); T. Duong et al., Microelectron. Eng. 67-68, 905 (2003).

[6] H. Risken, The Fokker-Planck Equation (Springer, Berlin, 1989), 2nd ed. 
(a)

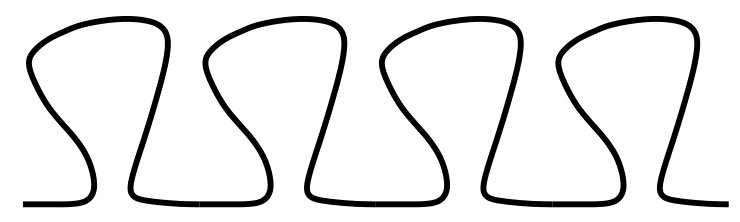

(b)

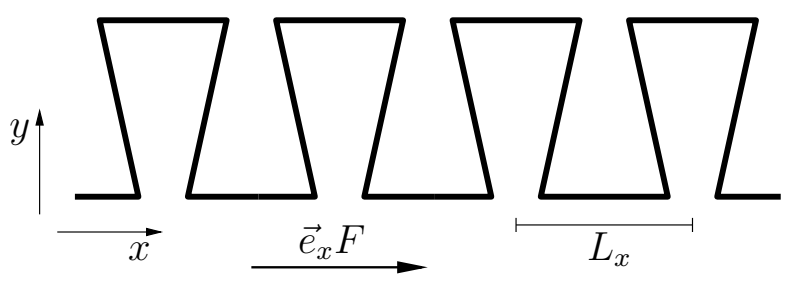

FIG. 1: Examples of quasi-one-dimensional meandering paths in the $x$ - $y$-plane (e.g. generated by a potential which is zero in the black and infinity in the white domains). They are required to be periodic (with period $L_{x}$ ) along the $x$-axis and confined along the $y$-axis. A further essential feature is the existence of sharp bends such that a steady motion along the meandering path results in a back-and-forth motion when projected along the $x$-axis. In general, no further symmetry is required, as exemplified with (a). The piecewise linear example (b) of high symmetry serves to simplify the discussion of the basic physical mechanisms. A homogeneous, static bias force $F$ is applied along the $x$-direction. (a)

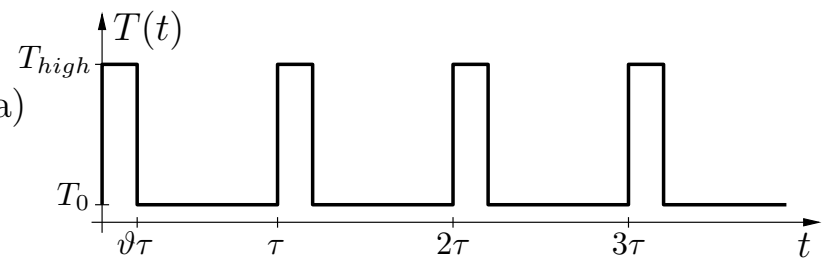

(b)

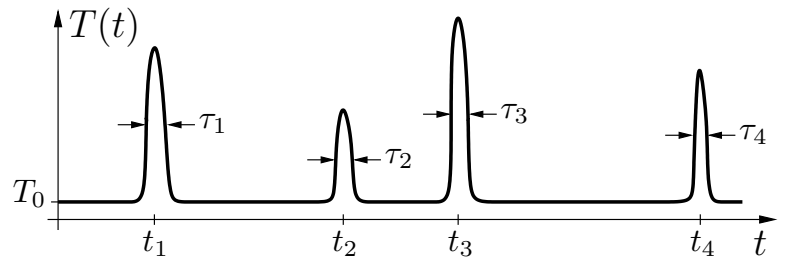

FIG. 2: Examples of time-dependent temperatures $T(t)$. (a) $T(t)$ is periodically switching between $T_{h i g h}$ and $T_{0}$. The total period is $\tau$ and the duration of the high- and low-temperature segments $\vartheta \tau$ and $(1-\vartheta) \tau$, respectively, with $\vartheta \in(0,1)$. (b) $T(t)$ consist of a "background" $T_{0}$ and random "spikes" at times $t_{i}$ of duration $\tau_{i}$. Their average distance and duration are characterized according to Eqs. (1), (2). 
(a)

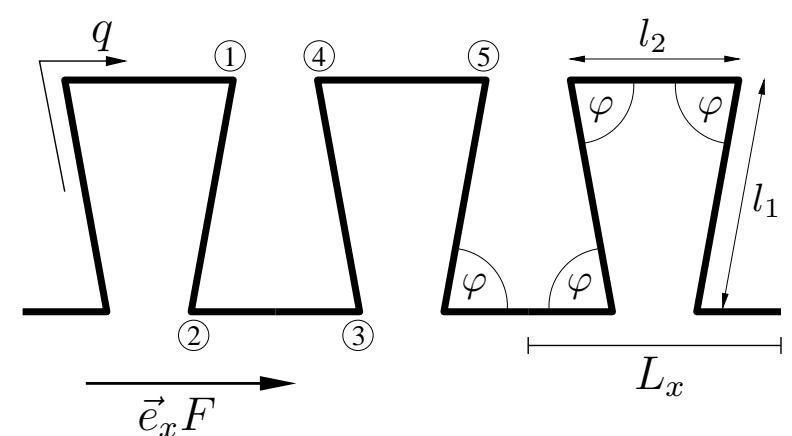

(b)

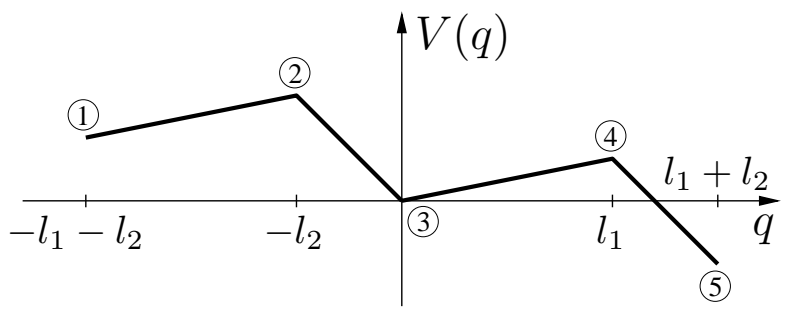

FIG. 3: (a) Same example as in Fig. 1b, parametrized by the angle $\varphi$ and the lengths $l_{1}, l_{2}$. (b) The corresponding onedimensional potential $V(q)$ as described above Eq. (5), i.e., $V^{\prime}(q)=-F$ for $q \in\left[-l_{2}, 0\right]$ or $q \in\left[l_{1}, l_{1}+l_{2}\right]$ and $V^{\prime}(q)=$ $F \cos \varphi$ for $q \in\left[-l_{1}-l_{2},-l_{2}\right]$ or $q \in\left[0, l_{1}\right]$. The encircled numbers mark corresponding positions in (a) and (b).

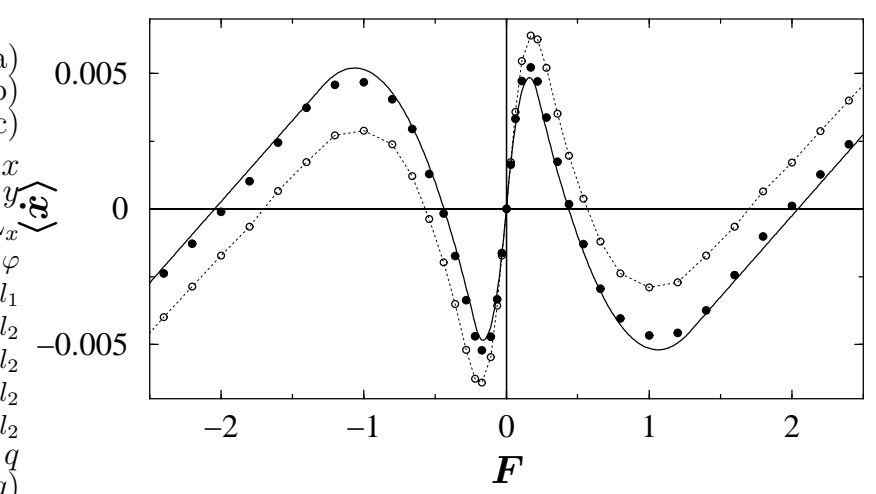

FIG. 4: Average particle current (3) versus static force $F$ for the meandering path in Fig. 3a with $l_{1}=3, l_{2}=2$, $\varphi=75^{\circ}$, and a time-periodic temperature according to Fig. 2a with $\tau=10$, and $\vartheta=0.1$. Filled circles: $T_{0}=0.001$, $T_{\text {high }}=50$. Open circles: $T_{0}=0.01, T_{\text {high }}=5$. Shown are results from numerical simulations of (4)-(11) in dimensionless units with $\eta=k_{B}=1$. The numerical uncertainty is about the symbol size. Solid line: The theoretical approximation (15), coinciding with (16) since $T(t)$ is periodic. Dotted line: guide for the eye. 


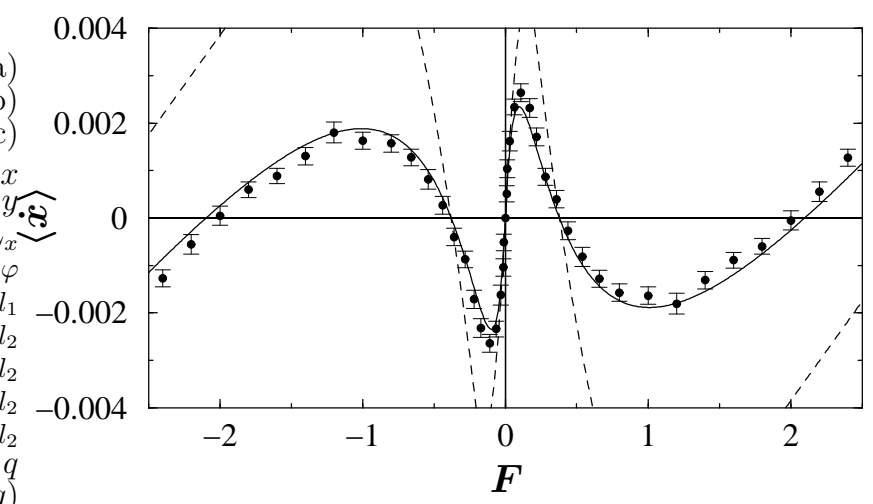

FIG. 5: Filled circles: Same as in Fig. 4 with the exception that the spiking times $t_{i}$ are not periodic [as in Fig. 2a] but rather randomly sampled [as in Fig. 2b] according to a Poisson process with average interspike distance $\tau=10$ [cf. (11)]. The relative duration $\tau_{i}=0.07=\vartheta$ is the same for all spikes $i$ [cf. (2)]. Solid line: Theoretical approximation (15). Dashed line: Simplified approximation (16). 